\title{
The Pólya-Szegö Principle and the Anisotropic Convex Lorentz-Sobolev Inequality
}

\author{
Shuai Liu and Binwu He \\ Department of Mathematics, Shanghai University, Shanghai 200444, China \\ Correspondence should be addressed to Binwu He; hebinwu@shu.edu.cn
}

Received 11 May 2014; Revised 24 June 2014; Accepted 24 June 2014; Published 15 July 2014

Academic Editor: Seyed S. Seyedalizadeh

Copyright ( 92014 S. Liu and B. He. This is an open access article distributed under the Creative Commons Attribution License, which permits unrestricted use, distribution, and reproduction in any medium, provided the original work is properly cited.

An anisotropic convex Lorentz-Sobolev inequality is established, which extends Ludwig, Xiao, and Zhang's result to any norm from Euclidean norm, and the geometric analogue of this inequality is given. In addition, it implies that the (anisotropic) Pólya-Szegö principle is shown.

\section{Introduction}

The classical Pólya-Szegö principle (see, e.g., $[1,2])$ states that for $p \geq 1$ the inequality

$$
\int_{\mathbb{R}^{n}}|\nabla f|^{p} d x \geq \int_{\mathbb{R}^{n}}\left|\nabla f^{\star}\right|^{p} d x
$$

holds for every $f \in C_{0}^{\infty}\left(\mathbb{R}^{n}\right)$, where $C_{0}^{\infty}\left(\mathbb{R}^{n}\right)$ denotes the set of functions on $\mathbb{R}^{n}$ that are smooth and have compact support and $|\cdot|$ is the standard Euclidean norm. Here $f^{\star}$ denotes the Schwarz symmetrization of $f$, that is, a function whose level sets have the same measure as the level sets of $f$ and are dilates of the Euclidean unit ball $B$. It has important applications to a large class of variational problems in different areas, for example, isoperimetric inequalities, optimal forms of Sobolev inequalities, and sharp a priori estimates of solutions to second-order elliptic or parabolic boundary value problems.

An anisotropic version of the classical Pólya-Szegö principle has been proved in [3], where convex symmetrization of $f$ is involved, which states that if $K$ is an origin-symmetric compact convex set, then for $p \geq 1$ the inequality

$$
\int_{\mathbb{R}^{n}}\|\nabla f\|_{K^{o}}^{p} d x \geq \int_{\mathbb{R}^{n}}\left\|\nabla f^{K}\right\|_{K^{o}}^{p} d x
$$

holds for every $f \in C_{0}^{\infty}\left(\mathbb{R}^{n}\right)$, where $\|\cdot\|_{K^{o}}$ is the Minkowski functional of the polar body of $K$. Here $f^{K}$ denotes the convex symmetrization of $f$, that is, a function whose level sets have the same measure as the level sets of $f$ and are dilates of the set $K$. Obviously, (2) reduces to (1) when $K=B$ (see Section 2 for unexplained notation and terminology).

A new approach to understanding Pólya-Szegö principle was proposed recently by Lutwak et al. [4] and Zhang [5]. Instead of using the classical technique on level sets $[f]_{t}$, their approach is using the $L^{p}$ convexification of level sets $\langle f\rangle_{t}$. This technique plays a fundamental role in the newly emerged affine Pólya-Szegö principle (see, e.g., [4-9]). Despite this progress, the study of the Pólya-Szegö principle by using this technique is vacancy. This is the motivation of the present paper. More precisely, we show the Pólya-Szegö principle from the $L^{p}$ Brunn-Minkowski theory, different from the known proofs of the Pólya-Szegö principle based on the geometric measure theory (see, e.g., $[1-3,10-16]$ ).

In [17], Ludwig et al. proved the following convex Lorentz-Sobolev inequality (see Theorem 2 in [17]): if $f \in$ $C_{0}^{\infty}\left(\mathbb{R}^{n}\right)$ and $1 \leq p<n$, then

$$
\int_{\mathbb{R}^{n}}|\nabla f|^{p} d x \geq n \kappa_{n}^{p / n} \int_{0}^{\infty} V\left(\langle f\rangle_{t}\right)^{(n-p) / n} d t,
$$

where $V$ denotes the Lebesgue measure on $\mathbb{R}^{n}$ with $\kappa_{n}=$ $V(B)=\pi^{n / 2} / \Gamma(1+n / 2)$. This inequality has a geometric analogue, namely, the following $L^{p}$ isoperimetric inequality: for $1<p<n$,

$$
S_{p}(L) \geq n \kappa_{n}^{p / n} V(L)^{(n-p) / n},
$$


where $L$ is an origin-symmetric compact convex set in $\mathbb{R}^{n}$ and $S_{p}(L)$ is the $L^{p}$ surface area of $L$.

In this paper we establish the following anisotropic convex Lorentz-Sobolev inequality.

Theorem 1. If $f \in C_{0}^{\infty}\left(\mathbb{R}^{n}\right), 1 \leq p \neq n$, and $K$ is an originsymmetric convex body in $\mathbb{R}^{n}$, then

$$
\int_{\mathbb{R}^{n}}\|\nabla f\|_{K^{o}}^{p} d x \geq n V(K)^{p / n} \int_{0}^{\infty} V\left(\langle f\rangle_{t}\right)^{(n-p) / n} d t
$$

with equality if and only if $\langle f\rangle_{t}$ is a dilate of $K$ for almost every $t>0$.

Similarly, our inequality (5) has a geometric analogue, namely, the following $L^{p}$ Minkowski inequality, for $1<p<$ $n$

$$
V_{p}(L, K) \geq V(L)^{(n-p) / n} V(K)^{p / n}
$$

where $L, K$ are origin-symmetric compact convex sets in $\mathbb{R}^{n}$ and $V_{p}(L, K)$ is the $L^{p}$ mixed volume of $L, K$.

When $L=B$, from $S_{p}(L)=n V_{p}(L, B),(5)$ and (6) reduce to (3) and (4), respectively.

It is shown that our inequality (5) implies the anisotropic Pólya-Szegö principle (2) for $1 \leq p \neq n$ in Theorem 5. Hence it is also true in Euclidean case; that is, (3) implies (1) for $1 \leq$ $p \neq n$. The arguments after Theorem 5 yield the fact that the anisotropic Pólya-Szegö principle (2) is still true for $p=n$ if we use the solution to the even normalized $L^{p}$ Minkowski problem.

\section{Background Material}

2.1. Elements of the $L^{p}$ Brunn-Minkowski Theory. For later reference, we quickly recall in this subsection some background material from the $L^{p}$ Brunn-Minkowski theory of convex bodies. This theory has its origin in the work of Firey from the 1960s and has expanded rapidly over the last couple of decades (see, e.g., [4, 8, 18-33]).

A convex body is a compact convex set in $\mathbb{R}^{n}$ which is throughout assumed to contain the origin in its interior. We denote by $\mathscr{K}_{o}^{n}$ the space of convex bodies equipped with the Hausdorff metric. Each convex body $K$ is uniquely determined by its support function $h_{K}=h(K, \cdot): \mathbb{R}^{n} \rightarrow \mathbb{R}$ defined by

$$
h_{K}(x)=h(K, x):=\max \{x \cdot y: y \in K\} .
$$

Let $\|\cdot\|_{K}: \mathbb{R}^{n} \rightarrow[0, \infty)$ denote the Minkowski functional of $K \in \mathscr{K}_{o}^{n}$; that is, $\|x\|_{K}=\min \{\lambda \geq 0: x \in \lambda K\}$. by

The polar set $K^{o}$ of $K \in \mathscr{K}_{o}^{n}$ is the convex body defined

$$
K^{o}=\left\{x \in \mathbb{R}^{n}: x \cdot y \leq 1 \forall y \in K\right\} .
$$

If $K \in \mathscr{K}_{o}^{n}$, then it follows from the definitions of support functions and Minkowski functionals, as well as the definition of polar body, that

$$
h_{K}(\cdot)=h(K, \cdot)=\|\cdot\|_{K^{o}} .
$$

For $p \geq 1, K, L \in \mathscr{K}_{o}^{n}$, the $L^{p}$ Minkowski combination $K+{ }_{p} L$ is the convex body defined by

$$
h\left(K+{ }_{p} L, \cdot\right)^{p}=h(K, \cdot)^{p}+h(L, \cdot)^{p} .
$$

The $L^{p}$ mixed volume $V_{p}(K, L)$ of $K, L \in \mathscr{K}_{o}^{n}$ is defined in $[25]$ by

$$
V_{p}(K, L)=\frac{p}{n} \lim _{\varepsilon \rightarrow 0^{+}} \frac{V\left(K+{ }_{p} \varepsilon^{1 / p} L\right)-V(K)}{\varepsilon} .
$$

In particular,

$$
V_{p}(K, K)=V(K)
$$

for every convex body $K$.

It was shown in [25] that, for all convex bodies $K, L \in \mathscr{K}_{o}^{n}$,

$$
V_{p}(K, L)=\frac{1}{n} \int_{S^{n-1}} h_{L}^{p}(u) d S_{p}(K, u),
$$

where $S_{p}(K, u)=h_{K}(u)^{1-p} d S(K, u)$ and the measure $S(K, \cdot)$ on $S^{n-1}$ is the classical surface area measure of $K$. Recall that, for a Borel set $\omega \subset S^{n-1}, S(K, \omega)$ is the $(n-1)$-dimensional Hausdorff measure of the set of all boundary points of $K$ for which there exists a normal vector of $K$ belonging to $\omega$.

Note that

$$
S_{p}(t K, \cdot)=t^{n-p} S_{p}(K, \cdot)
$$

for all $t>0$ and convex bodies $K$.

2.2. The Convex Symmetrization of Functions. Given any measurable function $f: \mathbb{R}^{n} \rightarrow \mathbb{R}$ such that $V\left(\left\{x \in \mathbb{R}^{n}\right.\right.$ : $|f(x)|>t\})<\infty$ for every $t>0$, its distribution function $\mu_{f}:[0, \infty) \rightarrow[0, \infty]$ is defined by

$$
\mu_{f}(t)=V\left(\left\{x \in \mathbb{R}^{n}:|f(x)|>t\right\}\right) .
$$

The decreasing rearrangement $f^{*}:[0, \infty) \rightarrow[0, \infty]$ of $f$ is defined by

$$
f^{*}(s)=\inf \left\{t \geq 0: \mu_{f}(t) \leq s\right\} .
$$

The Schwarz symmetrization of $f$ is the function $f^{\star}: \mathbb{R}^{n} \rightarrow$ $[0, \infty]$ defined by

$$
f^{\star}(x)=f^{*}\left(\kappa_{n}|x|^{n}\right),
$$

where $|\cdot|$ is the standard Euclidean norm.

For an origin-symmetric convex body $K$, the convex symmetrization $f^{K}$ of $f$ with respect to $K$ is defined as follows:

$$
f^{K}(x)=f^{*}\left(\kappa_{n}\|x\|_{\widetilde{K}}^{n}\right),
$$

where $\|x\|_{\widetilde{K}}$ is the Minkowski functional of $\widetilde{K}$, with $\widetilde{K}$ being a dilate of $K$ so that $V(\widetilde{K})=\kappa_{n}$. Note that $f, f^{*}$, and $f^{K}$ are equimeasurable; that is,

$$
\mu_{f}=\mu_{f^{*}}=\mu_{f^{K}} .
$$


Therefore, we have

$$
\|f\|_{\infty}=f^{*}(0)=\left\|f^{K}\right\|_{\infty} .
$$

We will frequently apply Federer's co-area formula (see, e.g., [34, page 258]). We state a version which is sufficient for our purposes: if $f: \mathbb{R}^{n} \rightarrow \mathbb{R}$ is Lipschitz and $g: \mathbb{R}^{n} \rightarrow$ $[0, \infty)$ is measurable, then, for any Borel set $A \subseteq \mathbb{R}$,

$$
\int_{f^{-1}(A) \cap\{|\nabla f|>0\}} g(x) d x=\int_{A} \int_{f^{-1}\{t\}} \frac{g(x)}{|\nabla f(x)|} d \mathscr{H}^{n-1}(x) d t,
$$

where $\mathscr{H}^{n-1}$ denotes $(n-1)$-dimensional Hausdorff measure.

2.3. The $L^{p}$ Convexification of Level Sets. Suppose $f \in$ $C_{0}^{\infty}\left(\mathbb{R}^{n}\right)$. For each real $t>0$, define the level set

$$
[f]_{t}=\left\{x \in \mathbb{R}^{n}:|f(x)| \geq t\right\} .
$$

By Sard's theorem, for almost every $t>0$, the boundary

$$
\partial[f]_{t}=\left\{x \in \mathbb{R}^{n}:|f(x)|=t\right\}
$$

of $[f]_{t}$ is a smooth $(n-1)$-dimensional submanifold of $\mathbb{R}^{n}$ with everywhere nonzero normal vector $\nabla f(x)$.

Now, we explain the technique called the $L^{p}$ convexification of level sets (see [17] for more details). Let $f: U \rightarrow \mathbb{R}$, where $U \subset \mathbb{R}^{n}$ is open, be locally Lipschitz; let $t>0$; and suppose $\nabla f(x) \neq 0$ for almost everywhere on $\partial[f]_{t}=\{x \in$ $U:|f(x)|=t\}$. For $1 \leq p \neq n$, define the $L^{p}$ convexification $\langle f\rangle_{t}$ of the level set $[f]_{t}$ as the unique origin-symmetric convex body such that

$$
\int_{S^{n-1}} \varphi(u) d S_{p}\left(\langle f\rangle_{t}, u\right)=\int_{\partial[f]_{t}} \varphi(\nu(x))|\nabla f|^{p-1} d \mathscr{H}^{n-1}(x)
$$

for all even $\varphi \in C\left(S^{n-1}\right)$, where $\nu(x)=-\nabla f(x) /|\nabla f(x)|$.

Thus, equality (24) holds for almost every $t>0$ if $f \in$ $C_{0}^{\infty}\left(\mathbb{R}^{n}\right)$.

\section{The Anisotropic Convex Lorentz-Sobolev Inequality}

The following lemma can be proved in the spirit of $[17,31$, 35](e.g., see Lemma 3 in [35]).

Lemma 2. If $f \in C_{0}^{\infty}\left(\mathbb{R}^{n}\right)$ and $K, L$ are origin-symmetric convex bodies, then, for almost every $t \in\left(0,\|f\|_{\infty}\right)$ and $1 \leq$ $p \neq n,\left\langle f^{K}\right\rangle_{t}$ is a dilate of $K$ and

$$
V\left(\left\langle f^{K}\right\rangle_{t}\right)=V\left(\left\langle f^{L}\right\rangle_{t}\right)
$$

Proof. Since $h_{K^{o}}$ is Lipschitz (and therefore differentiable almost everywhere) and $h_{K^{o}}(x)=1$ on $\partial K$, then, for almost every $x \in \partial K$,

$$
v_{K}(x)=\frac{\nabla h_{K^{o}}(x)}{\left|\nabla h_{K^{o}}(x)\right|}
$$

where $v_{K}(x)$ is the outer unit normal vector of $K$ at the point $x$. Note that $h_{K}\left(\nabla h_{K^{o}}(x)\right)=1$, for almost every $x \in \mathbb{R}^{n}$; hence we have

$$
h_{K}\left(\nu_{K}(x)\right)=\frac{1}{\left|\nabla h_{K^{o}}(x)\right|}
$$

Since $f^{K}$ is Lipschitz, then, for almost every $t \in\left(0,\|f\|_{\infty}\right)$, the set $\partial\left[f^{K}\right]_{t}$ is the boundary of a dilate of $K$ with nonvanishing normal $\nabla f^{K}$. It follows from Sard's theorem that

$$
\mathscr{H}^{n}\left(\left\{x \in \mathbb{R}^{n}:|f(x)|=t\right\}\right)=0 \quad \text { for almost every } t>0 \text {. }
$$

Hence there exists a unique $s>0$ such that $t=f^{*}\left(\kappa_{n} s^{n}\right)$ for almost every $t \in\left(0,\|f\|_{\infty}\right)$. Indeed, we have $s=\left(\mu_{f}(t) / \kappa_{n}\right)^{1 / n}$. Then by (24), (18), (9), and the fact that $\nabla h_{\widetilde{K}^{o}}$ is homogeneous of degree 0 and (27), we obtain that

$$
\begin{aligned}
& \int_{S^{n-1}} \varphi^{p}(u) d S_{p}\left(\left\langle f^{K}\right\rangle_{t}, u\right) \\
& =\int_{\partial\left[f^{K}\right]_{t}} \varphi^{p}(\nu(x))\left|\nabla f^{K}(x)\right|^{p-1} d \mathscr{H}^{n-1}(x) \\
& =\int_{\partial\left[f^{K}\right]_{t}} \varphi^{p}(\nu(x)) \\
& \times\left|\left(f^{*}\right)^{\prime}\left(\kappa_{n} h_{\widetilde{K}^{o}}(x)^{n}\right) n \kappa_{n} h_{\widetilde{K}^{o}}(x)^{n-1} \nabla h_{\widetilde{K}^{o}}(x)\right|^{p-1} d \mathscr{H}^{n-1}(x) \\
& =\int_{s \partial \widetilde{K}} \varphi^{p}(\nu(x))\left|\left(f^{*}\right)^{\prime}\left(\kappa_{n} s^{n}\right) n \kappa_{n} s^{n-1} \nabla h_{\widetilde{K}^{o}}(x)\right|^{p-1} d \mathscr{H}^{n-1}(x) \\
& =s^{n-1}\left(-\left(f^{*}\right)^{\prime}\left(\kappa_{n} s^{n}\right) n \kappa_{n} s^{n-1}\right)^{p-1} \\
& \times \int_{\partial \widetilde{K}} \varphi^{p}\left(\nu_{\widetilde{K}}(x)\right)\left|\nabla h_{\widetilde{K}^{o}}(x)\right|^{p-1} d \mathscr{H}^{n-1}(x) \\
& =\left(\frac{\mu_{f}(t)}{\kappa_{n}}\right)^{(n-1) / n}\left(-\left(f^{*}\right)^{\prime}\left(\mu_{f}(t)\right) n \kappa_{n}\left(\frac{\mu_{f}(t)}{\kappa_{n}}\right)^{(n-1) / n}\right)^{p-1} \\
& \times \int_{\partial \widetilde{K}} \varphi^{p}\left(\nu_{\widetilde{K}}(x)\right) h_{\widetilde{K}}\left(\nu_{\widetilde{K}}(x)\right)^{1-p} d \mathscr{H}^{n-1}(x) \\
& =n^{p-1} \kappa_{n}^{(p-n) / n} \mu_{f}(t)^{(n-1) p / n}\left(-\mu_{f}^{\prime}(t)\right)^{1-p} \\
& \times \int_{S^{n-1}} \varphi^{p}(u) d S_{p}(\widetilde{K}, u)
\end{aligned}
$$

for almost every $t \in\left(0,\|f\|_{\infty}\right)$ and even $\varphi \in C\left(S^{n-1}\right)$. Thus, the uniqueness of the solution of the even $L_{p}$ Minkowski problem [25] and (14) implies that

$$
\left\langle f^{K}\right\rangle_{t}=c(f, t)^{1 /(n-p)} \widetilde{K} \quad \text { for almost every } t \in\left(0,\|f\|_{\infty}\right),
$$


where $c(f, t)=n^{p-1} \kappa_{n}^{(p-n) / n} \mu_{f}(t)^{(n-1) p / n}\left(-\mu_{f}^{\prime}(t)\right)^{1-p}$. Since $f^{c K}=f^{K}$ for any $c>0$ and any $K \in \mathscr{K}_{o}^{n}$, we have

$$
V\left(\left\langle f^{K}\right\rangle_{t}\right)=V\left(\left\langle f^{L}\right\rangle_{t}\right)=c(f, t)^{n /(n-p)} \kappa_{n}
$$

for almost every $t \in\left(0,\|f\|_{\infty}\right)$.

Recall that the $L^{p}$ Minkowski inequality [25] states the following.

Theorem 3. If $p \geq 1$ and $L, K \in \mathscr{K}_{o}^{n}$, then

$$
V_{p}(L, K) \geq V(L)^{1-p / n} V(K)^{p / n}
$$

with equality if and only if $L, K$ are dilates when $p>1$ and if and only if $L, K$ are homothetic when $p=1$.

Now, we prove the anisotropic convex Lorentz-Sobolev inequality.

Proof of Theorem 1 . Noting that $h_{K}(\cdot)=\|\cdot\|_{K^{o}}$, by the co-area formula (21), (24), (13), and (32), we have

$$
\begin{aligned}
\int_{\mathbb{R}^{n}} h_{K}(\nabla f)^{p} d x & =\int_{0}^{\infty} \int_{\partial[f]_{t}} h_{K}(\nabla f)^{p} \frac{1}{|\nabla f|} d \mathscr{H}^{n-1}(x) d t \\
& =\int_{0}^{\infty} \int_{\partial[f]_{t}} h_{K}(\nu(x))^{p}|\nabla f|^{p-1} d \mathscr{H}^{n-1}(x) d t \\
& =\int_{0}^{\infty} \int_{S^{n-1}} h_{K}(u)^{p} d S_{p}\left(\langle f\rangle_{t}, u\right) d t \\
& =\int_{0}^{\infty} n V_{p}\left(\langle f\rangle_{t}, K\right) d t \\
& \geq n V(K)^{p / n} \int_{0}^{\infty} V\left(\langle f\rangle_{t}\right)^{(n-p) / n} d t
\end{aligned}
$$

where $\nu(x)=-\nabla f(x) /|\nabla f(x)|$ on $\partial[f]_{t}$ for almost every $t>0$ and the second equality holds since $K$ is an origin-symmetric and the support function of $K$ is homogeneous of degree 1.

Equality (5) follows from equality (32) and the fact that $\langle f\rangle_{t}$ is origin-symmetric.

It is shown above, Proof of Theorem 1 , that the $L^{p}$ Minkowski inequality (32) implies inequality (5).

In what follows we will show that the $L^{p}$ Minkowski inequality (32) can be easily deduced from the anisotropic convex Lorentz-Sobolev inequality (5) for $1<p<n$ by taking

$$
f(x)=g\left(\|x\|_{L}\right), \quad \text { where } g(s)=\left(1+s^{p /(p-1)}\right)^{1-n / p} .
$$

Indeed, as shown in [17, Lemma 8],

$$
\langle f\rangle_{t}=c_{p}(t) L
$$

and $c_{p}(t)^{n-p}=\left|g^{\prime}(s)\right|^{p-1} s^{n-1}$ with $t=g(s)$. Hence

$$
\begin{aligned}
\int_{\mathbb{R}^{n}}\|\nabla f\|_{K^{o}}^{p} d x & =\int_{0}^{\infty} n V_{p}\left(\langle f\rangle_{t}, K\right) d t \\
& =\int_{0}^{\infty} n V_{p}\left(c_{p}(t) L, K\right) d t \\
& =n V(L, K) \int_{0}^{\infty} c_{p}(t)^{n-p} d t, \\
\int_{0}^{\infty} V\left(\langle f\rangle_{t}\right)^{(n-p) / n} d t & =\int_{0}^{\infty} V\left(c_{p}(t) L\right)^{(n-p) / n} d t \\
& =V(L)^{(n-p) / n} \int_{0}^{\infty} c_{p}(t)^{n-p} d t,
\end{aligned}
$$

where

$$
\int_{0}^{\infty} c_{p}(t)^{n-p} d t=\frac{(n-p)^{p}}{(p-1)^{p-1} p} B\left(\frac{n-p}{p}, \frac{n p-n+p}{p}\right) .
$$

\section{The Pólya-Szegö Principle}

The following theorem can be seen as a weak form of the Pólya-Szegö principle (2).

Theorem 4. If $f \in C_{0}^{\infty}\left(\mathbb{R}^{n}\right)$ and $K, L$ are origin-symmetric convex body such that $L$ is not a dilate of $K$, then, for $1 \leq p \neq n$,

$$
\int_{\mathbb{R}^{n}}\left\|\nabla f^{L}\right\|_{K^{o}}^{p} d x>\int_{\mathbb{R}^{n}}\left\|\nabla f^{K}\right\|_{K^{o}}^{p} d x .
$$

Proof. Since $\left\langle f^{L}\right\rangle_{t}$ is a dilate of $L$ for almost every $t \in$ $\left(0,\|f\|_{\infty}\right)$ by Lemma 2 , then the $L^{p}$ Minkowski inequality (32) between $\left\langle f^{L}\right\rangle_{t}$ and $K$ is strict for almost every $t \in$ $\left(0,\|f\|_{\infty}\right)$. Combined with (25), it follows that

$$
\begin{aligned}
\int_{\mathbb{R}^{n}}\left\|\nabla f^{L}\right\|_{K^{o}}^{p} d x & =\int_{0}^{\infty} n V_{p}\left(\left\langle f^{L}\right\rangle_{t}, K\right) d t \\
& >n V(K)^{p / n} \int_{0}^{\infty} V\left(\left\langle f^{L}\right\rangle_{t}\right)^{(n-p) / n} d t \\
& =n V(K)^{p / n} \int_{0}^{\infty} V\left(\left\langle f^{K}\right\rangle_{t}\right)^{(n-p) / n} d t \\
& =\int_{0}^{\infty} n V_{p}\left(\left\langle f^{K}\right\rangle_{t}, K\right) d t \\
& =\int_{\mathbb{R}^{n}}\left\|\nabla f^{K}\right\|_{K^{o}}^{p} d x .
\end{aligned}
$$

We are now in the position to prove the Pólya-Szegö principle (2).

Theorem 5. Suppose $K$ is an origin-symmetric convex bodies in $\mathbb{R}^{n}$. If $f \in C_{0}^{\infty}\left(\mathbb{R}^{n}\right), 1 \leq p \neq n$, then

$$
\int_{\mathbb{R}^{n}}\|\nabla f\|_{K^{o}}^{p} d x \geq \int_{\mathbb{R}^{n}}\left\|\nabla f^{K}\right\|_{K^{o}}^{p} d x .
$$


Proof. It was shown in $[4,(6.3)]$ that the following differential inequality holds:

$$
V\left(\langle f\rangle_{t}\right)^{(n-p) / n} \geq n^{p-1} \mu_{f}(t)^{(n-1) p / n}\left(-\mu_{f}^{\prime}(t)\right)^{1-p} .
$$

Integrating both sides of the inequality gives

$$
\begin{aligned}
& \int_{0}^{\infty} V\left(\langle f\rangle_{t}\right)^{(n-p) / n} d t \\
& \quad \geq n^{p-1} \int_{0}^{\infty} \mu_{f}(t)^{(n-1) p / n}\left(-\mu_{f}^{\prime}(t)\right)^{1-p} d t .
\end{aligned}
$$

Noting that $h_{K}(\cdot)=\|\cdot\|_{K^{o}}$ and Combined with (5), we obtain that

$$
\begin{aligned}
\int_{\mathbb{R}^{n}} & h_{K}(\nabla f)^{p} d x \\
& \geq n^{p} V(K)^{p / n} \int_{0}^{\infty} \mu_{f}(t)^{(n-1) p / n}\left(-\mu_{f}^{\prime}(t)\right)^{1-p} d t .
\end{aligned}
$$

By the homogeneous of $K$ in (43) and (40), we only need to consider $V(K)=\kappa_{n}$. So it is sufficient to prove that

$$
\int_{\mathbb{R}^{n}} h_{K}\left(\nabla f^{K}\right)^{p} d x=n^{p} \kappa_{n}^{p / n} \int_{0}^{\infty} \mu_{f}(t)^{(n-1) p / n}\left(-\mu_{f}^{\prime}(t)\right)^{1-p} d t .
$$

The last equality is shown in [3]. Now, we prove this equality by using Lemma 2 . Together with the co-area formula (21), the equality (24), the definition of $c(f, t)$ in Lemma 2, (13), and $V(K)=\kappa_{n}$, we obtain

$$
\begin{aligned}
\int_{\mathbb{R}^{n}} & h_{K}\left(\nabla f^{K}(x)\right)^{p} d x \\
= & \int_{0}^{\infty}\left(\int_{\partial\left[f^{K}\right]_{t}} \frac{h_{K}\left(\nabla f^{K}(x)\right)^{p}}{\left|\nabla f^{K}(x)\right|} d \mathscr{H}^{n-1}(x)\right) d t \\
= & \int_{0}^{\infty} \int_{\partial\left[f^{K}\right]_{t}} h_{K}(\nu(x))^{p}\left|\nabla f^{K}\right|^{p-1} d \mathscr{H}^{n-1}(x) d t \\
= & \int_{0}^{\infty} \int_{S^{n-1}} h_{K}(u)^{p} d S_{p}\left(\left\langle f^{K}\right\rangle_{t}, u\right) d t \\
= & \int_{0}^{\infty} \int_{S^{n-1}} c(f, t) h_{K}(u)^{p} d S_{p}(K, u) d t \\
= & \int_{0}^{\infty} c(f, t) d t \int_{S^{n-1}} h_{K}(u)^{p} d S_{p}(K, u) \\
= & n V(K) \int_{0}^{\infty} n^{p-1} \kappa_{n}^{(p-n) / n} \mu_{f}(t)^{(n-1) p / n}\left(-\mu_{f}^{\prime}(t)\right)^{1-p} d t \\
= & n^{p} \kappa_{n}^{p / n} \int_{0}^{\infty} \mu_{f}(t)^{(n-1) p / n}\left(-\mu_{f}^{\prime}(t)\right)^{1-p} d t,
\end{aligned}
$$

where $v(x)=-\nabla f^{K}(x) /\left|\nabla f^{K}(x)\right|$ on $\partial\left[f^{K}\right]_{t}$ for almost every $t>0$. And the second equality holds since $K$ is originsymmetric and the support function of $K$ is homogeneous of degree 1.
Moreover, Theorem 5 can be proved for $p \geq 1$ by using the solution to the even normalized $L^{p}$ Minkowski problem as in $[7,9]$. More precisely, suppose $f \in C_{0}^{\infty}\left(\mathbb{R}^{n}\right)$, for $p \geq 1$, and define the normalized $L^{p}$ convexification $\overline{\langle f\rangle_{t}}$ as the unique origin-symmetric convex body such that

$$
\begin{gathered}
\frac{1}{V\left(\widetilde{\langle f\rangle_{t}}\right)} \int_{S^{n-1}} g(u) d S_{p}\left(\widetilde{\langle f\rangle_{t}}, u\right) \\
=\int_{\partial[f]_{t}} g(\nu(x))|\nabla f|^{p-1} d \mathscr{H}^{n-1}(x),
\end{gathered}
$$

for almost every $t>0$. By taking slight modifications in the proof of Theorem 1, we obtain

$$
\int_{\mathbb{R}^{n}} h_{K}(\nabla f)^{p} d x \geq n V(K)^{p / n} \int_{0}^{\infty} V\left(\widetilde{\langle f\rangle_{t}}\right)^{-p / n} d t
$$

Similar to the proof of Theorem 5, together with the observation in $[7,(4.22)]$ that

$$
V\left(\widetilde{\langle f\rangle_{t}}\right)^{-p / n} \geq n^{p-1} \mu_{f}(t)^{(n-1) p / n}\left(-\mu_{f}^{\prime}(t)\right)^{1-p}
$$

we also get (43). So Theorem 5 remains true for $p=n$.

\section{Conflict of Interests}

The authors declare that they have no conflict of interests.

\section{Authors' Contribution}

All the authors contributed equally to the paper. All the authors read and approved the final paper.

\section{Acknowledgments}

This work is supported in part by the National Natural Science Foundation of China (Grant no. 11371239), Shanghai Leading Academic Discipline Project (Project no. J50101), and the Research Fund for the Doctoral Programs of Higher Education of China (20123108110001).

\section{References}

[1] G. Pólya and G. Szegö, Isoperimetric Inequalities in Mathematical Physics, Princeton University Press, Princeton, NJ, USA, 1951.

[2] G. Talenti, "Best constant in Sobolev inequality," Annali di Matematica Pura ed Applicata. Serie Quarta, vol. 110, pp. 353372, 1976.

[3] A. Alvino, V. Ferone, and G. Trombetti, "Convex symmetrization and applications," Annales de l'Institut Henri Poincare C: Non Linear Analysis, vol. 14, no. 2, pp. 275-293, 1997.

[4] E. Lutwak, D. Yang, and G. Zhang, "Sharp affine $L_{p}$ Sobolev inequalities," Journal of Differential Geometry, vol. 62, no. 1, pp. 17-38, 2002.

[5] G. Zhang, "The affine Sobolev inequality," Journal of Differential Geometry, vol. 53, no. 1, pp. 183-202, 1999. 
[6] D. Alonso-Gutiérrez, J. Bastero, and J. Bernués, "Factoring Sobolev inequalities through classes of functions," Proceedings of the American Mathematical Society, vol. 140, no. 10, pp. 35573566, 2012.

[7] A. Cianchi, E. Lutwak, D. Yang, and G. Zhang, "Affine MoserTrudinger and MORrey-Sobolev inequalities," Calculus of Variations and Partial Differential Equations, vol. 36, no. 3, pp. 419436, 2009.

[8] C. Haberl and F. E. Schuster, "Asymmetric affine $L_{p}$ Sobolev inequalities," Journal of Functional Analysis, vol. 257, no. 3, pp. 641-658, 2009.

[9] C. Haberl, F. Schuster, and J. Xiao, "An asymmetric affine PólyaSzegö principle," Mathematische Annalen, vol. 352, no. 3, pp. 517-542, 2012.

[10] J. E. Brothers and W. P. Ziemer, "Minimal rearrangements of Sobolev functions," Journal für die Reine und Angewandte Mathematik, vol. 384, pp. 153-179, 1988.

[11] A. Cianchi, L. Esposito, N. Fusco, and T. Trombetti, "A quantitative Pólya-Szegö principle," Journal für die Reine und Angewandte Mathematik, vol. 614, pp. 153-189, 2008.

[12] A. Cianchi and N. Fusco, "Functions of bounded variation and rearrangements," Archive for Rational Mechanics and Analysis, vol. 165, no. 1, pp. 1-40, 2002.

[13] L. Esposito and P. Ronca, "Quantitative Pólya-Szegö principle for convex symmetrization," Manuscripta Mathematica, vol. 130, no. 4, pp. 433-459, 2009.

[14] L. Esposito and C. Trombetti, "Convex symmetrization and Pólya-Szegö inequality," Nonlinear Analysis: Theory, Methods \& Applications, vol. 56, no. 1, pp. 43-62, 2004.

[15] A. Ferone and R. Volpicelli, "Minimal rearrangements of Sobolev functions: a new proof," Annales de l'Institut Henri Poincare (C): Non Linear Analysis, vol. 20, no. 2, pp. 333-339, 2003.

[16] A. Ferone and R. Volpicelli, "Convex rearrangement: equality cases in the Pólya-Szegö inequality," Calculus of Variations and Partial Differential Equations, vol. 21, no. 3, pp. 259-272, 2004.

[17] M. Ludwig, J. Xiao, and G. Zhang, "Sharp convex LorentzSobolev inequalities," Mathematische Annalen, vol. 350, no. 1, pp. 169-197, 2011.

[18] S. Campi and P. Gronchi, "The $L^{p}$-Busemann-Petty centroid inequality," Advances in Mathematics, vol. 167, no. 1, pp. 128-141, 2002.

[19] S. Campi and P. Gronchi, "On the reverse $L^{p}$-Busemann-Petty centroid inequality," Mathematika, vol. 49, no. 1-2, pp. 1-11 (2004), 2002.

[20] C. Haberl and F. E. Schuster, "General $\mathrm{L}_{p}$ affine isoperimetric inequalities," Journal of Differential Geometry, vol. 83, no. 1, pp. 1-26, 2009.

[21] D. Hug, E. Lutwak, D. Yang, and G. Zhang, "On the $L_{p}$ Minkowski problem for polytopes," Discrete \& Computational Geometry, vol. 33, no. 4, pp. 699-715, 2005.

[22] M. Ludwig, "Ellipsoids and matrix-valued valuations," Duke Mathematical Journal, vol. 119, no. 1, pp. 159-188, 2003.

[23] M. Ludwig, "Minkowski valuations," Transactions of the American Mathematical Society, vol. 357, no. 10, pp. 4191-4213, 2005.

[24] M. Ludwig and M. Reitzner, "A classification of $S L(n)$ invariant valuations," Annals of Mathematics, vol. 172, no. 2, pp. 1219-1267, 2010.

[25] E. Lutwak, "The Brunn-Minkowski-Firey theory. I. Mixed volumes and the Minkowski problem," Journal of Differential Geometry, vol. 38, no. 1, pp. 131-150, 1993.
[26] E. Lutwak, "The Brunn-Minkowski-Firey theory. II. Affine and geominimal surface areas," Advances in Mathematics, vol. 118, no. 2, pp. 244-294, 1996.

[27] E. Lutwak, D. Yang, and G. Zhang, " $L_{p}$ affine isoperimetric inequalities," Journal of Differential Geometry, vol. 56, no. 1, pp. 111-132, 2000.

[28] E. Lutwak, D. Yang, and G. Zhang, "A new ellipsoid associated with convex bodies," Duke Mathematical Journal, vol. 104, no. 3, pp. 375-390, 2000.

[29] E. Lutwak, D. Yang, and G. Zhang, "On the $\mathrm{L}_{p}$-Minkowski problem," Transactions of the American Mathematical Society, vol. 356, no. 11, pp. 4359-4370, 2004.

[30] E. Lutwak, D. Yang, and G. Zhang, " $L_{p}$ John ellipsoids," Proceedings of the London Mathematical Society, vol. 90, no. 2, pp. 497-520, 2005.

[31] E. Lutwak, D. Yang, and G. Zhang, "Optimal Sobolev norms and the $L^{p}$ Minkowski problem," International Mathematics Research Notices, vol. 2006, 21 pages, 2006.

[32] E. Werner and D. Ye, "New $L_{p}$ affine isoperimetric inequalities," Advances in Mathematics, vol. 218, no. 3, pp. 762-780, 2008.

[33] E. Werner and D. Ye, "Inequalities for mixed p-affine surface area," Mathematische Annalen, vol. 347, no. 3, pp. 703-737, 2010.

[34] H. Federer, Geometric Measure Theory, Springer, Berlin, Germany, 1969.

[35] T. Wang, "The affine Pólya-Szegö principle: equality cases and stability," Journal of Functional Analysis, vol. 265, no. 8, pp. 17281748, 2013. 


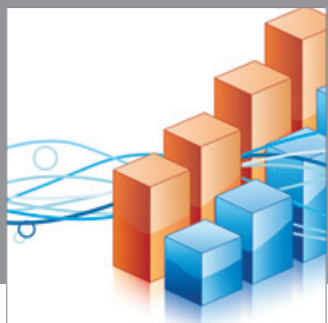

Advances in

Operations Research

mansans

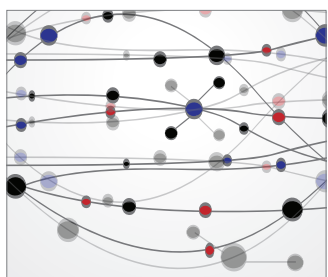

The Scientific World Journal
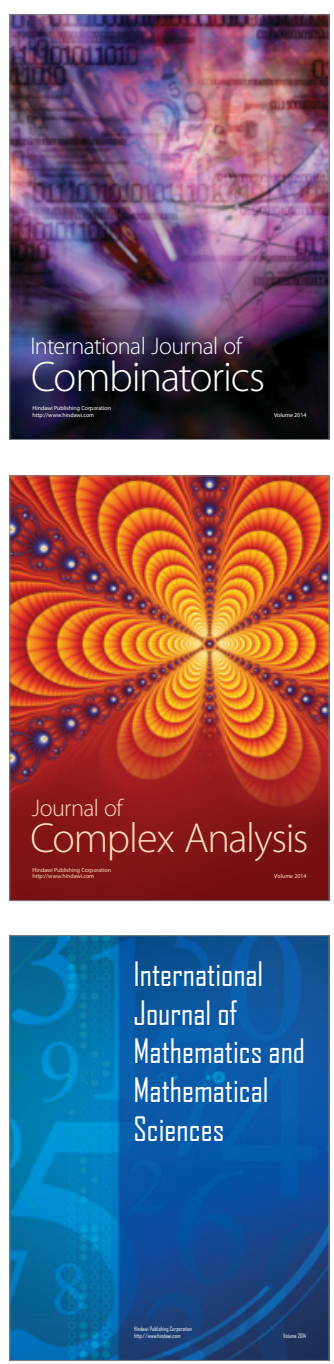
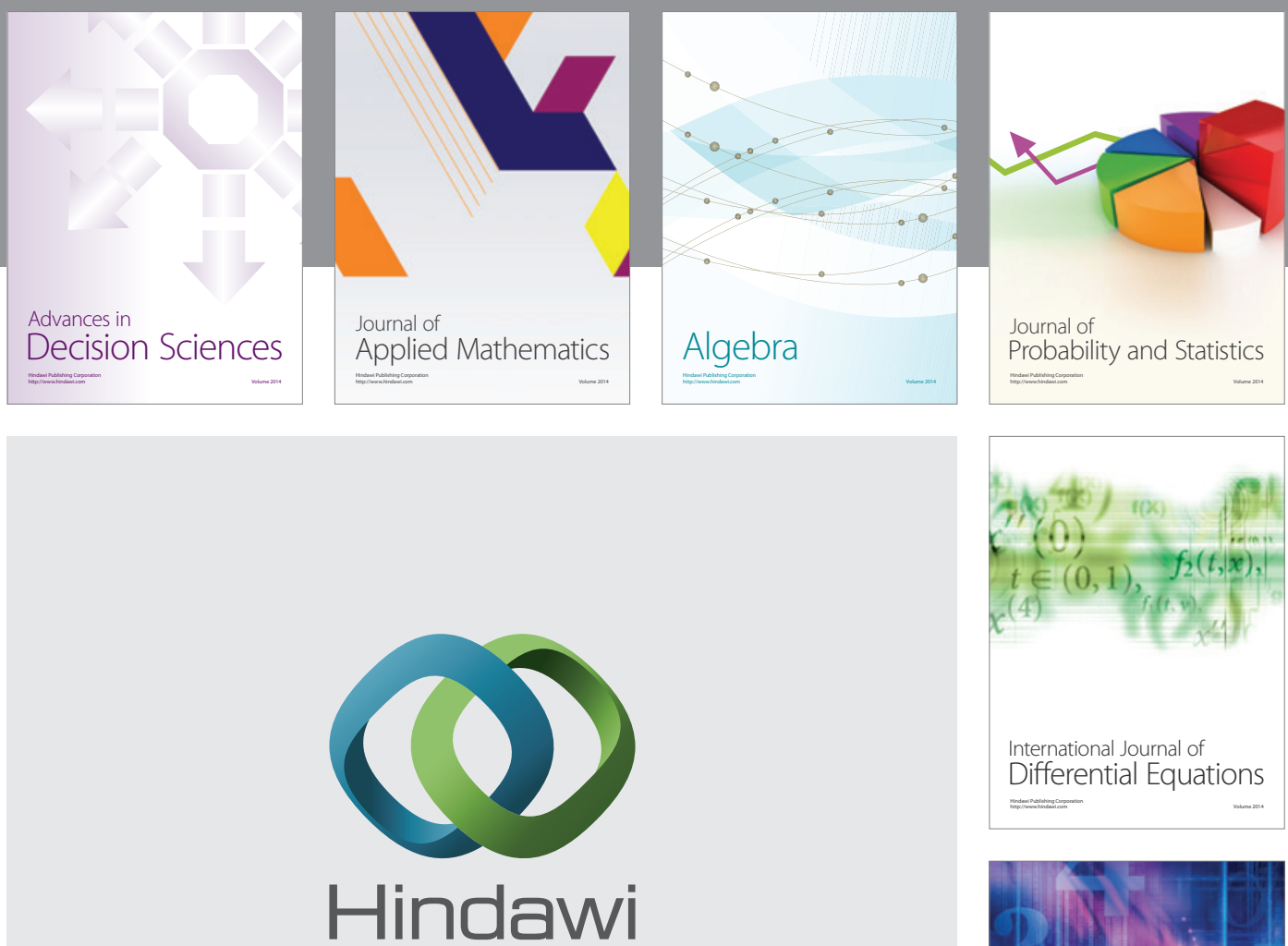

Submit your manuscripts at http://www.hindawi.com
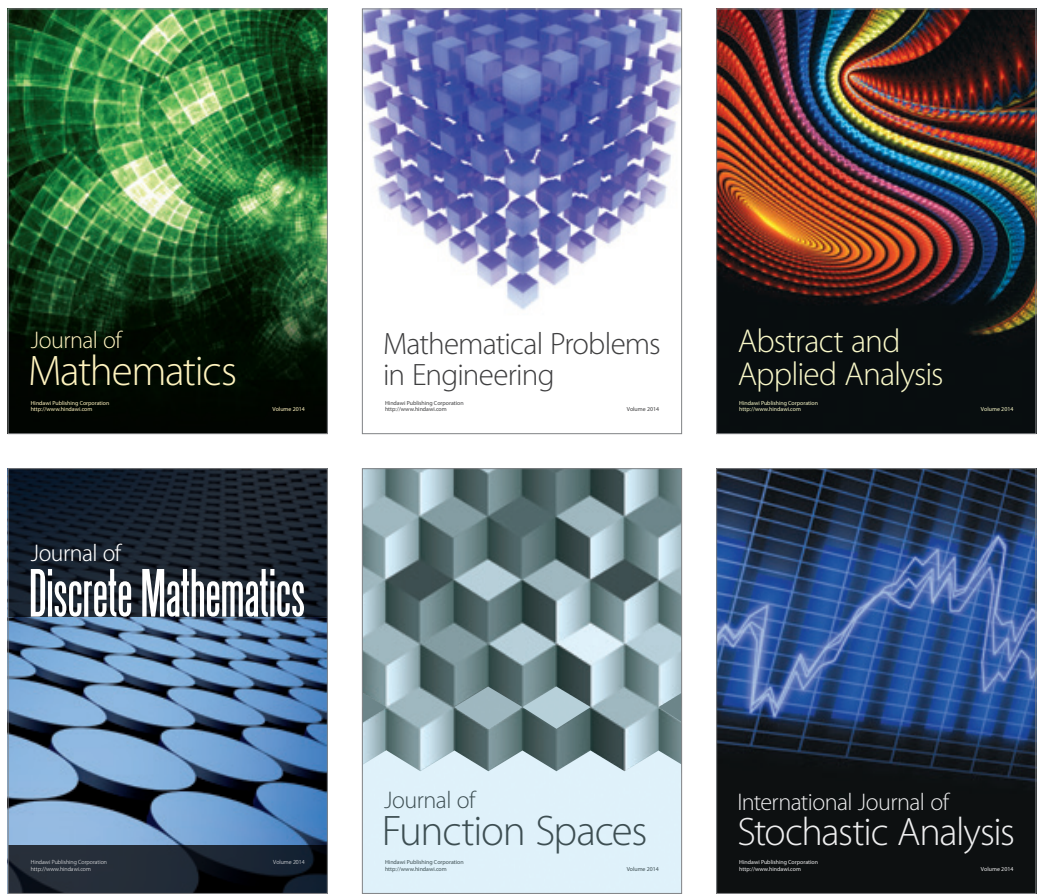

Journal of

Function Spaces

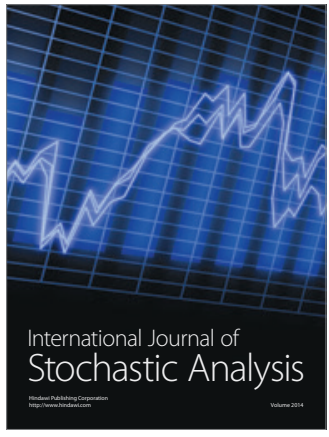

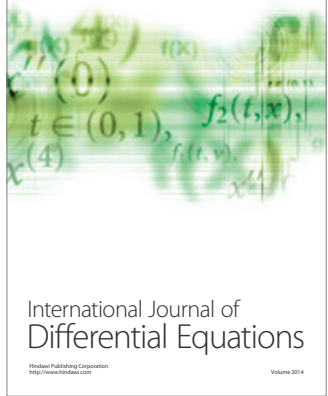
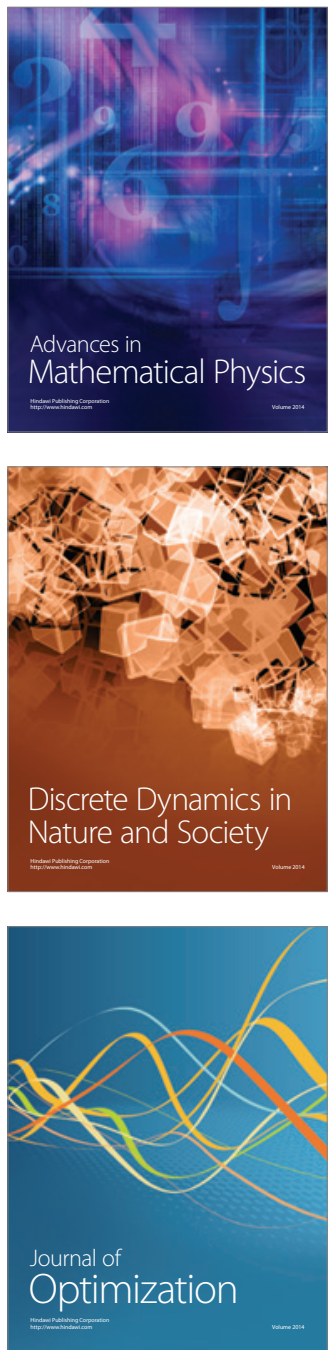